\title{
Monika Kurath
}

\section{Nichtwissen lenken}

Nanotechnologie in Europa und den Vereinigten Staaten 
Wissenschaft und Technik sind wesentliche Antriebskräfte gesellschaftlicher Veränderung. Sie haben in den letzten Jahrzehnten zugleich tief greifende Kontroversen und Konflikte über die Folgen und die möglichen Grenzen wissenschaftlicher und technischer Modernisierungsprozesse hervorgerufen. Daher berühren sie das Selbstverständnis moderner Gesellschaften in grundlegender Weise.

Die Reihe Wissenschafts- und Technikforschung widmet sich den gesellschaftlichen, organisatorischen und interaktiven Dimensionen moderner Wissenschaft und Technik, ihrem historischen Wandel, den Diskursen und Deutungsmustern, in denen sie kommuniziert und legitimiert werden, sowie den mit ihnen verbundenen ethischen und politischen Herausforderungen. Sie integriert Theorien und Methoden aus unterschiedlichen thematisch relevanten Disziplinen, vor allem aus Soziologie und Geschichtswissenschaft. Damit bietet sie sowohl Grundlagenwissen für die beteiligten wissenschaftlichen Disziplinen als auch Orientierungswissen für Entscheidungsträger und die interessierte Öffentlichkeit.

Schriftenreihe

„Wissenschafts- und Technikforschung"

herausgegeben von

Prof. Dr. Alfons Bora, Universität Bielefeld

Prof. Dr. Sabine Maasen, Universität Basel

Prof. Dr. Carsten Reinhardt, Universität Bielefeld

PD Dr. Peter Wehling, Universität Frankfurt am Main

\section{Band 18}


Monika Kurath

\section{Nichtwissen lenken}

Nanotechnologie in Europa und den Vereinigten Staaten 
Publiziert mit Unterstützung des Schweizerischen Nationalfonds zur Förderung der wissenschaftlichen Forschung.

Die Druckvorstufe dieser Publikation wurde vom Schweizerischen Nationalfonds zur Förderung der wissenschaftlichen Forschung unterstützt.

Die Deutsche Nationalbibliothek verzeichnet diese Publikation in der Deutschen Nationalbibliografie; detaillierte bibliografische Daten sind im Internet über http://dnb.d-nb.de abrufbar.

Zugl.: Wien, Univ., Habil, 2016

ISBN 978-3-8487-3636-2 (Print)

ISBN 978-3-8452-7969-5 (ePDF)

1. Auflage 2016

(c) Nomos Verlagsgesellschaft, Baden-Baden 2016. Gedruckt in Deutschland. Alle Rechte, auch die des Nachdrucks von Auszügen, der fotomechanischen Wiedergabe und der Übersetzung, vorbehalten. Gedruckt auf alterungsbeständigem Papier. 
Dieses Buch stellt den Abschluss einer langjährigen Auseinandersetzung dar, in welcher ich der Frage nachgegangen bin, wie Wissensgesellschaften mit Spitzentechnologien umgehen. Ich habe das Projekt im Jahr 2006 mit einem Stipendium des Schweizerischen Nationalfonds (SNF) am Programm für Wissenschaftsforschung der Universität Basel angefangen. Mit der Ambition, Regulierungsdiskurse der Nanotechnologie in Deutschland, Großbritannien, den USA und auf EU-Ebene zu untersuchen, habe ich mich auf eine Untersuchung eingelassen, deren Komplexität eher auf einem Forschungsverbund angelegt gewesen wäre, als auf ein Einzelprojekt. Während dreier Jahre habe ich 74 qualitative Interviews geführt, sowie mehr als 160 Regulierungsdokumente und über 50 Lenkungsmaßnahmen untersucht. Das Projekt hat mich bei Forschungsaufenthalten am ,Program for Science, Technology \& Society' an der Harvard Kennedy School und am Institut für Wissenschafts- und Technikforschung an der Universität Wien begleitet. Es ist Gegenstand von mehr als 30 Konferenzbeiträgen, Präsentationen und Publikationen. Seine Niederschrift hat mit Unterbrechungen sechs Jahre und bis zu seinem endgültigen Abschluss als Buch ein weiteres Jahr gedauert.

Damit hat es sich endgültig vom Status eines einfachen Forschungsprojektes verabschiedet und ist zur Lebensabschnittsaufgabe geworden. In dieser Phase, in welcher ich an fünf verschiedenen Forschungseinrichtungen tätig war, zwei neue Forschungsbereiche aufgebaut, eine Forschungsgruppenleitung übernommen und zwei weitere Kinder bekommen habe, waren mir zahlreiche Mentorinnen und Förderer, Kolleginnen und Kollegen sowie Freunde und Familie eine unabdingbare Stütze. Es ist mir ein Anliegen, ihnen nachfolgend sehr herzlich zu danken.

Wenn diese Arbeit einen ,spiritus rector' kennt, so kommt diese Rolle Sabine Maasen zu. Sie hat damals am Programm für Wissenschaftsforschung der Universität Basel die Nanotechnologie als logische Fortsetzung meiner Forschungsinteressen identifiziert und nie aufgehört, daran zu glauben und mich zu ermuntern, dass die Arbeit doch noch zu einem Ende findet. Ohne ihre stete Ermahnung, den Fall nicht aus den Augen zu verlieren, hätte ich es kaum geschafft, die Nanotechnologie als Nichtwissensfrage zu identifizieren. 
Ulrike Felt hat sich nach dem Weggang von Sabine Maasen aus Basel entscheidend dafür eingesetzt, dass ich mich an der Universität Wien habilitieren konnte. Mit dem Angebot einer Gastprofessur habe ich die Möglichkeit erhalten, eine wunderbare Zeit in Wien zu verbringen. Mit den sympathischen Kolleginnen und Kollegen am Institut für Wissenschaftsund Technikforschung habe ich inspirierende Diskussionen geführt und mit einer Gruppe äußerst wacher Studierenden Wien erkundet und Ansätze der Wissenschafts- und Technikforschung auf die Untersuchung von Stadt und Stadtplanung angewendet. Ganz herzlich danke ich Uli für ihre langjährige wohlwollende Begleitung meiner Forschung und das angenehme und kollegiale Habilitationsverfahren.

Max Fochler bleibt mir ein Vorbild in Gelassenheit. Dank seiner freundlichen und hoch professionellen Einführung ins Institut und in die Lehre, habe ich kaum bemerkt, dass ich den Ort gewechselt und in einem anderen kulturellen Kontext unterrichtet habe. Nach unserer gemeinsamen Zeit in Harvard war es eine besondere Freude, das Büro erneut mit Eric Aarden zu teilen. Ihm, Max, Dorothea Born, Jasmin Engelhart, Nikolaus Pöchhacker und Katharina Simma danke ich herzlich für ihre kollegiale Unterstützung in der Habilitationsphase. In bester Erinnerung bleibt mir Alan Irwin. Mit seinem einmaligen Humor, mit welchem er wissenschaftliche Beiträge ebenso wie Begegnungen auf Nebenschauplätzen kommentiert, hat er uns alle bestens unterhalten.

Die Fertigstellung dieses Buches entscheidend unterstützt haben Julia Nentwich und Chris Steyaert vom Institut für Organisationspsychologie an der Universität St.Gallen (OPSY) mit ihrem Geschenk eines ruhigen Büroraums. Die Kolleginnen und Kollegen am OPSY haben mich in der Endphase der Habilitation freundschaftlich begleitet, insbesondere Mohammed Shafiullah, der während seiner Abwesenheit seinen Schreibtisch so großzügig an mich abgetreten hat, Christoph Michels mit seinem vorbildlichen Optimismus und Katharina Molterer und Mark Laukamm, die mich zur Mittagszeit stets so charmant vom Computer weggelockt haben.

Tanja Schneider, die ich nach unserer gemeinsamen Zeit am Basler Programm für Wissenschaftsforschung in St.Gallen wiedergetroffen habe und Julia Nentwich, mit der mich seit unserer Dissertationszeit in St. Gallen vielfältige gemeinsame Interessen verbinden, haben mir im Endspurt des Habilitationsverfahrens unschätzbare moralische und intellektuelle Unterstützung geleistet. Ebenfalls in diese Phase fällt die Unterstützung, die ich von Franz Schultheis und Rolf Wüstenhagen bei der Entwicklung eines weiterführenden Forschungsprojektes erhalten habe. 
Meine Kolleginnen und Kollegen am ETH Wohnforum - ETH CASE haben mein Forschungsprojekt stets großzügig unterstützt, obwohl es so ganz und gar nicht in den Forschungsfokus des Instituts gepasst hat. Dennoch haben mich Dietmar Eberle, Margrit Hugentobler und Marie Glaser stets darin bestärkt, das Projekt zu einem glücklichen Ende zu bringen. Den produktiven Austausch insbesondere mit Ignaz Strebel, Jan Silberberger und Krishna Bharathi habe ich sehr geschätzt. Mit meinen Doktorandinnen und Doktoranden, Anna Flach, Marko Marskamp, Julio Paulos und Bernhard Böhm erlebe ich eine großartige Zusammenarbeit, in welcher wir Ambition mit Spaß verbinden und so Höchstleistungen erbringen. Mit viel Toleranz und Gelassenheit halten sie eine Betreuerin aus, die ebenfalls mitten in der akademischen Beförderungs- und Berufungsphase steckt. Dafür können sie nicht genug gelobt werden.

Meine Kolleginnen und Kollegen am Programm für Wissenschaftsforschung haben mich in meiner frühen Postdocphase moralisch unterstützt und intellektuell herausgefordert. Mit Mario Kaiser verbindet mich eine langjährige Forschungspartnerschaft in den empirischen Tiefen der Nanotechnologie, aus welchen wir beide zwar spät aber dennoch gutgelaunt wieder aufgetaucht sind. Mit Andreas Lösch verbindet mich ebenfalls ein langjähriger stimulierender Austausch. Arie Rip hat mir wichtige methodische Hinweise gegeben.

In Basel hatte ich auch das Glück, dem bestgelauntesten Gastwissenschaftler aller Zeiten - Alexander Bogner - zu begegnen. Seit seinem legendären Open-Air-Vortrag im Sommer 2008, hat Alex zu vielfältigen Sternstunden an unterschiedlichen wissenschaftlichen Veranstaltungen beigetragen und gezeigt, dass Spaß eine unabdingbare Voraussetzung von Exzellenz darstellt. Damit hat er nicht nur meine Forschungslaufbahn, sondern auch meine Haltung dazu entscheidend geprägt.

Sheila Jasanoff vom Program for Science, Technology and Society an der Harvard Kennedy School hat mir gezeigt, was echte Nachwuchsförderung bedeutet und welche Verantwortung Vorgesetzte für ihre wissenschaftlichen Mitarbeitenden tragen. Mit ihrem unermüdlichen Einsatz, diversesten Gruppen von Gastforschenden die Wissenschafts- und Technikforschung näherzubringen und uns intellektuell zu Höchstleistungen anzuspornen, hat sie mein wissenschaftliches Denken und Arbeiten entscheidend geprägt. Rob Hagendijk hat mir mit seinen hilfreichen Kommentaren und entscheidenden Hinweisen den Partizipationsdiskurs in der Wissenschafts- und Technikforschung veranschaulicht. Meine Kolleginnen und Kollegen am Fellow-Programm, insbesondere Eric Aarden, Iris Eisenber- 
ger, Sang-Hyun Kim und Martyn Pickersgill haben dieser Arbeit mit ihren hilfreichen Kommentaren in den Forschungskolloquien wichtige Impulse gegeben und auch den Freizeitwert meines Neuenglandaufenthalts bereichert.

Gerd Folkers und Johannes Fehr haben mir nach meiner Stipendienzeit am Collegium Helveticum mit dem Angebot eines Arbeitsplatzes in den architektonisch einmaligen Räumen der Sempersternwarte ruhiges Arbeiten und interdisziplinären Austausch ermöglicht, was für das Gelingen dieser Arbeit äußerst wertvoll war. Mit Priska Gisler verbindet mich eine langjährige Forschungspartnerschaft. Priska hat mich in die Methoden des qualitativen Interviews eingeführt und damit einen zentralen Grundstein für mein wissenschaftliches Arbeiten gelegt. Martina Merz begleitet diese Arbeit ebenfalls seit ihren Anfängen und hat meine Forschung durch unseren produktiven Austausch über gemeinsame Forschungsinteressen stets vielfältig unterstützt. Ebenfalls produktive Begleiter meiner Forschung waren Michael Guggenheim, der mir auch das Kochen für Großgruppen beigebracht, Rainer Egloff, der mich nicht nur mit dem Gedankengut Ludwik Flecks bekannt gemacht, sondern auch immer wieder musikalisch überrascht und Christian Pohl, der mich in die interdisziplinäre Lehre an der ETH Zürich eingeführt hat. Helga Nowotny danke ich dafür, dass sie mich als meine erste Mentorin in die Wissenschafts- und Technikforschung eingeführt und damit den Grundstein für eine anhaltende Leidenschaft und Begeisterung für das Fach gelegt hat.

Sehr herzlich danke ich all meinen Interviewpartnerinnen und Interviewpartner, die mit ihrer Geduld und Bereitschaft, mir Auskunft zu geben, diese Arbeit überhaupt erst möglich gemacht haben. Ein besonderer Verdienst kommt hier Andy Hofmann zu, der mich mit Gwen Ruta von Environmental Defense bekannt gemacht hat, die mir den Zugang zu wesentlichen Akteuren des Nanotechnologiediskurses in den USA eröffnet hat. Antje Grobe von Dialog Basis, Hans Kastenholz damals Empa und Torsten Fleischer vom KIT-ITAS verdanke ich vielfältige Hinweise zum Partizipations- und politischen Diskurs der Nanotechnologie in Deutschland. Der Austausch mit Sergio Bellucci von TA Swiss hat meine Arbeit ebenfalls befruchtet. Bei der Transkription der Interviews unterstützt haben mich Beate Luber und Naomi Lubick.

Für seine Unterstützung im sprachlichen Feinschliff dieser Arbeit danke ich Uwe Hamschmidt. Seiner kompromisslosen Forderung, dass Sätze nicht mehr als 30 Wörter beinhalten dürfen, ist es zu verdanken, dass diese Arbeit überhaupt lesbar geworden ist. Meiner Familie; meinen Eltern, Ver- 
wandten und Nachbarn danke ich sehr herzlich für ihre vielfältige Unterstützung, ohne die meine Kombination von Wissenschaft und Familie nie so produktiv möglich gewesen wäre. Meinem Mann, Jost Hamschmidt, danke ich für das partnerschaftliche Mittragen meiner Arbeit, für den anregenden Austausch, seinen Humor und seine Toleranz gegenüber den unterschiedlichen Phasen und Befindlichkeiten, die der Spagat zwischen Wissen-, Partner- und Elternschaft so mit sich bringt. Meinen Kindern Lena, Leo und Carlo schließlich danke ich sehr herzlich dafür, dass sie mich immer wieder produktiv ablenken, aus meinen Gedanken reißen und mich dazu zwingen, die wirklich wesentlichen Dinge des Lebens im Auge zu behalten. 
https://doi.org/10.5771/9783845279695-1

Generiert durch IP '172.22.53.54', am 26.04.2023, 14:46:57.

Das Erstellen und Weitergeben von Kopien dieses PDFs ist nicht zulässig. 
Inhaltsverzeichnis

Abkürzungsverzeichnis

1 Zur Soziologie des Nichtwissens

1.1 Die wissenssoziologische Analyse von

Nichtwissensdiskursen

1.2 Nichtwissen lenken $\quad 30$

1.3 Lenkungskulturen

1.4 Die Nanotechnologie als technowissenschaftliches Phänomen

1.5 Thesen und Fragestellung 45

$\begin{array}{ll}1.6 \text { Gliederung des Bandes } & 48\end{array}$

2 Abschätzen 51

2.1 Abschätzen in Nichtwissensdiskursen 54

2.2 Von Innovationen zu Implikationen, von der Technologie zum Material und von ELSI zu EHS

2.3 Vereinigte Staaten: Nationale Initiative, Visionen und soziale Innovationen

2.3.1 Eine neue ,Frontier'

2.3.2 Bedeutungverlust der Technikfolgenabschätzung

2.3.3 Wissenbasierte Ökonomie und die NNI 67

2.3.4 Soziale Implikationen als Innovationen 70

2.3.5 Beratungsgremien zum Innovationsmanagement

2.3.6 Von Innovationen zu Implikationen und von der Technologie zum Material

2.3.7 Von ELSI zu EHS

2.3.8 Abschätzung durch Umwelt-, Gesundheits- und Arbeitsschutzbehörden

2.3.9 Regulierungsfragen und EHS-Forschungsbedarf 
2.3.11 Vereinigte Staaten: Innovationen und ausgewählte Implikationen

2.4 Europäische Union: Innovationen und Implikationen in Strategie und Aktionsplan

2.4.1 Integration von Implikationen in Strategie und Aktionsplan

2.4.2 Ethische Fragen und strategische Evaluation

2.4.3 Regulierungsfragen

2.4.4 Implikationsabschätzung in nichtnanotechnologiespezifischen Gremien

2.4.5 Eine Technikfolgenabschätzung fürs Parlament

2.4.6 Europäische Union: Integration in die bestehenden Strukturen

2.5 Großbritannien: Implikationen statt Innovationen und Expertengremien

2.5.1 Forschungsexzellenz ohne nationale Förderinitiative

2.5.2 Implikationen als zentraler Fokus

2.5.3 Die Festschreibung des Diskurses durch die Royal Society

2.5.4 Beratungsgremien für den Umgang mit Implikationen

2.5.5 Forschungslücken und Regulierungsfragen

2.5.6 Lebensmittel- und Kosmetika

2.5.7 Eine nationale Nanotechnologiestrategie

2.5.8 Großbritannien: Implikationsorientierter Abschätzungsdiskurs

2.6 Deutschland: Aktionsplan für Innovationen und TA für Implikationen

2.6.1 Positionen und Aktionspläne des

Forschungsministeriums

2.6.2 Technikfolgenabschätzung

2.6.3 Definitionen und von der Technologie zum Material 134

2.6.4 Lebensmittel und Verbraucherschutz

2.6.5 Umwelt- und Gesundheitsfragen

2.6.6 Regulatorische Implikationen

2.6.7 Kooperation der Vorsorgebehörden

2.6.8 ,Hightech-Strategie' und EHS Forschungsbedarf

2.6.9 Neuer Aktionsplan 2015 
2.6.10 Deutschland: Hightech, EHS- und regulatorische Implikationen

2.7 Nichtwissen abschätzen: Gesellschaftlich distribuierte Expertise

3 Regulieren

3.1 Regulieren in Nichtwissensdiskursen 160

3.2 Vorsorge versus Vermeidung: Zwei Regulierungskulturen 165

3.2.1 Nanomaterialien zwischen Vorsorge und Vermeidung 170

3.2.2 Innovation versus Regulierung - ein Mythos? 174

3.2.3 Verschwimmende Regulierungskulturen durch internationale Kooperation

3.3 Vereinigte Staaten: Freiwilliges Datenmeldeverfahren und unübliche Kooperation

3.3.1 Das koordinierte Regelwerk des Nanotechnology Research and Development Act

3.3.2 Nanomaterialien in der Umwelt- und Chemikalienregulierung

3.3.3 Private Initiativen: Das EDF - DuPont ,Nano Risk Framework'

3.3.4 Zusammenfassung Vereinigte Staaten: Ein

Regulierungsdefizit und eine regierungsexterne Initiative

3.4 Europäische Union: REACH und ein Forschungskodex

3.4.1 Nanomaterialien und REACH

3.4.2 Freiwillige Maßnahmen: Empfehlungen der Kommission zu Forschung und Definition

3.4.3 Die gesetzliche Regulierung: Das sektorale Produktrecht

3.4.4 Zusammenfassung Europäische Union: Regulieren oder implementieren?

3.5 Großbritannien: Ein freiwilliges Datenmeldeverfahren und eine unübliche Kooperation

3.5.1 Nanomaterialien im Stoffrecht

3.5.2 Der Royal Society - Insight Investment Responsible Nano Code 


\subsubsection{Zusammenfassung Großbritannien: ein}

Regulierungsdefizit und eine Nichtregierungsinitiative 242

3.6 Deutschland: Evaluationen des Stoffrechts und selbstregulierende Hersteller

3.6.1 Nanomaterialien im Stoffrecht

3.6.2 Selbstregulierung durch Hersteller

3.6.3 Zusammenfassung Deutschland: Ein

Regulierungsdefizit und selbstregulierende Hersteller

3.7 Nichtwissen Regulieren: Gesellschaftliche Distribution der Regulierungsverantwortung

4.1 Partizipation in Nichtwissensdiskursen

4.2 Von der Aufklärung über den Dialog zum gleichberechtigten Austausch

4.2.1 Partizipation im Nanotechnologiediskurs

4.2.2 Kritische Reflexion der zivilgesellschaftlichen Partizipation

4.2.3 Wachsende Bedeutung der Anspruchsgruppenpartizipation

4.3 Vereinigte Staaten: Expertenpartizipation und Nichtregierungsinitiativen

4.3.1 Fehlende Einbindung von Anspruchsgruppen

4.3.2 Nichtregierungsinitiativen zur Anspruchsgruppenpartizipation

4.3.3 Information im Geiste von PUS statt Einbindung der Bevölkerung

4.3.4 Regierungsexterne zivilgesellschaftliche Partizipation: ASU und NISE

4.3.5 Zusammenfassung Vereinigte Staaten: Ein Partizipationsdefizit und Nichtregierungsinitiativen

4.4 Europäische Union: Partizipation als Strategie

4.4.1 Anspruchsgruppenpartizipation in Strategie und Aktionsplan

4.4.2 Anspruchsgruppenpartizipation als wissenschaftlicher Kongress und Forschungsprojekt 
4.4.3 Zivilgesellschaftliche Partizipation:

,Laborpartizipation' und Einweg-Kommunikation

4.4.4 Zusammenfassung Europäische Union: Partizipation als Forschung und Strategie

4.5 Großbritannien: Anspruchsgruppenkommissionen und ,upstream engagement'

4.5.1 Die Royal Society als Strategiebereiterin

4.5.2 Experten- und Steuerungsgremien für Anspruchsgruppenpartizipation

4.5.3 Regierungsexterne Initiativen der Anspruchsgruppenpartizipation

4.5.4 Zivilgesellschaftliche Partizipation als , upstream engagement'

4.5.5 Zusammenfassung Großbritannien: Expertengremien und zivilgesellschaftliche Partizipation als nationale Strategie

4.6 Deutschland: Nationaler Anspruchsgruppendialog und Herstellerinitiativen

4.6.1 Anspruchsgruppenpartizipation als

Regierungsaufgabe: die NanoKommission der

Bundesregierung

4.6.2 Nichtregierungsinitiativen der

Anspruchsgruppenpartizipation

333

4.6.3 Information der Zivilgesellschaft und

Meinungserhebung statt Einbindung

4.6.4 Zusammenfassung Deutschland:

Anspruchsgruppenpartizipation als

Regierungsprogramm

4.7 Nichtwissen externalisieren: Partizipationsparadox in

Nichtwissensdiskursen

5 Perspektiven für die Soziologie des Nichtwissens

5.1 Gesellschaftliche Phänomene in Nichtwissensdiskursen

5.2 Innovation, Integration, Implikation und Konsens: 4

Kulturen im Umgang mit Nichtwissen

5.2.1 Vereinigte Staaten: Innovation

5.2.2 Europäische Union: Integration 
5.2.3 Großbritannien: Implikationen $\quad 362$

5.2.4 Deutschland: Konsens 363

5.2.5 Vier Lenkungskulturen im Umgang mit Nichtwissen 364

5.3 Die Nicht-Lenkbarkeit von Nichtwissen 366

6 Literatur 371

7 Liste der Interviewpartner (anonymisiert) 401 


\section{Abkürzungsverzeichnis}

ACC

ACS

BAM

BAuA

BFR

BIS UK

BLL

BMBF

BMU

BRTF UK

BSE

BSI UK

BUND

CA EU

CAA US

CBEN

Cefic

CLP

CNS-ASU

CNS-UCSB

COSHH UK

CSA network

CST UK

CT US
American Chemistry Council

American Chemical Society

Deutsche Bundesanstalt für Materialforschung und -prüfung

Deutsche Bundesanstalt für Arbeitsschutz und Arbeitsmedizin

Deusches Bundesinstitut für Risikobewertung

Department for Business, Innovation and Skills

Deutscher Bund für Lebensmittelrecht und Lebensmittelkunde e.V.

Deutsches Bundesministerium für Bildung und Forschung

Deutsches Bundesministerium für Umwelt, Naturschutz und Reaktorsicherheit

Better Regulation Task Force

Bovine spongiforme Enzephalopathie

British Standards Institution

Bund für Umwelt und Naturschutz Deutschland; Friends of the Earth Germany

Competent Authorities

Clean Air Act

Center for Biological and Environmental Nanotechnology, Rice University, USA

European Chemical Industry Council

European Regulation on the Classification, Labelling and Packaging of substances and mixtures

Centre for Nanotechnology in Society, Arizona State University, USA

Centre for Nanotechnology in Society, University of California Santa Barbara, USA

Health and Safety Executives' Control of Substances Hazardous for Health

UK Chief Scientific Adviser Network

Council for Science and Technology

Committee on Technology 
CWA US

DEFRA UK

DG

DG-ENTR

DG-ENV

DG-RTD

DG-Sanco

DGUV

DH UK

DIUS UK

DOC US

DOD US

DOE US

DOH UK

DOL US

DTI UK

EA

ECAST

EDF

EEA

EGE

EHS

ELI

ELSI

EPA US

EPSRC UK

ESRC UK

ETC

FDA US
Clean Water Act

Department for Environment, Food and Rural Affairs

für Département Géneral bzw. Generaldirektion der Europäischen Kommission

Europäische Kommission: Generaldirektion Unternehmen und Industrie

Europäische Kommission: Generaldirektion Umwelt

Europäische Kommission: Generaldirektion Forschung und Innovation

Europäische Kommission: Generaldirektion Gesundheit und Verbraucher

Deutsche Gesetzliche Unfallversicherung

Department of Health, UK Department of Health

Department for Innovation, Universities and Skills

Department of Commerce

Department of Defense

Department of Energy

Department of Health

Department of Labor

Department for Trade and Industry

Europäische Akademie (Sitz in Deutschland)

Expert and Citizen Assessment of Science and Technology Network

Environmental Defense Fund, USA

European Environmental Agency

European Group on Ethics in Science and New Technologies

für englisch: Environment, Health and Safety; also Umwelt, Gesundheit und Sicherheit

Environmental Law Institute, USA

für englisch: Ethical, Legal and Social Implications, also ethische, rechtliche und soziale Implikationen

Environmental Protection Agency

Engineering and Physical Sciences Research Council

Economic and Social Research Council

Action Group on Erosion, Technology and Concentration

Food and Drug Administration 
FIFRA US

FSA UK

GAO US

HGP US

HLSTC UK

HSAC UK

HSE UK

ICCA

ICON

IOM

IÖW

IRC

IRGC

IS UK

ISO

IT

IWGN US

JRC

MHPRA UK

MRC UK

N\&N

nanobüro

Nanotechnology Nanotechnology Knowledge Transfer Network

KTN UK

NASA US

NCG UK

NCTF US

NEG UK

NEHI US

NIA UK
Federal Insecticide, Fungicide and Rhodendicide Act, U.S. Federal Insecticide, Fungicide and Rhodendicide Act

Food Standards Agency, UK Food Standards Agency

Government Accountability Office

Human Genome Project

House of Lords Science and Technology Committee

Advisory Committee on Hazardous Substances

Health and Safety Executive, UK Health and Safedy Executive

International Council of Chemical Associations

International Council on Nanotechnology

UK Institute for Occupational Medicine

Institut für Ökologische Wirtschaftsforschung, Berlin

Interdisciplinary Research Collaboration in Nanotechnology, UK

International Risk Governance Council

Department for Business, Innovation and Skills

Internationale Standardorganisation

Informationstechnologie

Interagency Working Group on Nanoscience, Engineering and Technology

European Commission Joint Research Center

Medicines and Healthcare Products Regulatory Agency

Medical Research Council

Auf Unionsebene verwendete Abkürzung für Nanowissenschaften und Nanotechnologien

Büro für Interdisziplinäre Nanotechnikforschung, TU Darmstadt, Deutschland

National Aeronautics and Space Administration

Nanotechnologies Collaboration Group

National Citizens' Technology Forum

Nanotechnology Engagement Group

Nanomaterial Environment and Health Implications working group

Nanotechnology Industries Association 


\begin{tabular}{|c|c|}
\hline NIDG UK & Nanotechnology Issues Dialogue Group \\
\hline NIJ US & National Institute of Justice \\
\hline NIMR UK & National Institute for Medical Research \\
\hline NION UK & National Initiative on Nanotechnology, \\
\hline NIOSH US & National Institutes of Occupationaly Safety and Health \\
\hline NISE Net & Nanoscale Informal Science Education Network USA \\
\hline NMSP & Nanomaterial Stewardship Program, EPA, USA \\
\hline NNAP US & National Nanotechnology Advisory Panel \\
\hline NNCO US & National Nanotechnology Coordination Office \\
\hline NNFC UK & National Nanotechnology Fabrication Centres \\
\hline NNI US & National Nanotechnology Initiative \\
\hline NNIN US & National Nanotechnology Infrastructure Network \\
\hline NNUN US & National Nanofabrication Users Network \\
\hline NPL UK & National Physical Laboratory \\
\hline NRC US & National Research Council \\
\hline NRCG UK & Nanotechnologies Research Coordination Group \\
\hline NRDA US & 21st Century Nanotechnology Research and Development Act \\
\hline NRF & $\begin{array}{l}\text { Environmental Defense Fund - Du Pont Nano Risk Framework, } \\
\text { USA }\end{array}$ \\
\hline NRSG UK & Nanotechnology Resarch Strategies Group \\
\hline NSET US & Nanoscale Science, Engineering and Technology Subcommittee \\
\hline NSF UK & $\begin{array}{l}\text { Nanotechnologies Stakeholder Forum, UK Nanotechnology Strat- } \\
\text { egy Forum, und U.S. National Science Foundation }\end{array}$ \\
\hline NSTC US & National Science and Technology Council \\
\hline OECD & $\begin{array}{l}\text { Organisation for Economic Co-operation and Development; } \\
\text { deutsch Organisation für wirtschaftliche Zusammenarbeit und Ent- } \\
\text { wicklung }\end{array}$ \\
\hline OSHA US & Occupational Safety and Health Administration \\
\hline OSI UK & Office of Science and Innovation \\
\hline OSTP US & Office of Science and Technology Policy \\
\hline OTA US & Office for Technology Assessment \\
\hline PCAST US & President's Council of Advisors on Science and Technology \\
\hline PEALS & Policy, Ethics and Life Sciences Research Centre, UK \\
\hline PEN & Project on Emerging Nanotechnologies, USA \\
\hline pTA & partizipative Technikfolgenabschätzung \\
\hline
\end{tabular}


PTB

PUS

RAE.

RCEP UK

RCRA US

RCUK

$\mathrm{REACH}$

$\mathrm{ReNaTe}$

RNC

RNF

RoHS

RS

RS\&RAE

SCCP EU

SCENIHR

SNUR

Sofia

STOA EU

STS

TA

TAB

TAG US

TSCA US

UBA

UfU

USDA US

VCI

VDI

VRS

vzbv
Deutsche Physikalisch-Technische Bundesanstalt

Public Understanding of Science

Royal Academy of Engineering, UK

Royal Commission on Environmental Pollution

Resource Conservation and Recovery Act

Research Councils UK

European Regulation on Registration, Evaluation Authorisation and Restriction of Chemicals

Rechtsgutachten Nanotechnologie, Deutschland

Responsible Nanocode, UK

Responsible Nano Forum, UK

European Regulation on the Restriction of Hazardous Substances

Royal Society, UK

Royal Society und Royal Academy of Engineering

Scientific Committee on Consumer Products

European Scientific Committee for Emerging and Newly Identified Risks

Significant New Use Rules unter dem U.S. Toxic Substance Control Act TSCA

Sonderforschungsgruppe Institutionenanalyse Darmstadt,

Deutschland

Parliament Science and Technology Options Assessment

Science and Technology Studies

Technikfolgenabschätzung

Büro für Technikfolgenabschätzung des Deutschen Bundestags

Technical Advisory Group

Toxic Substance Control Act

Deutsches Umweltbundesamt

Unabhängiges Institut für Umweltforschung e.V. Deutschland

Department of Acriculture

Verband der Chemischen Industrie e.V., Deutschland

Verein Deutscher Ingenieure

Voluntary Reporting Scheme for Engineered Nanoscale Materials, DEFRA, UK

Deutsche Verbraucherzentrale Bundesverband e.V. 
Abkürzungsverzeichnis

Wilson-Zentrum Woodrow Wilson International Center for Scholars, USA WPMN OECD-Working Party on Manufactured Nanomaterials

WPN OECD-Working Party on Nanotechnology

WTEC World Technology Evaluation Centre, USA 